\title{
The Connection Between Childhood Maltreatment and Eating Disorder Psychopathology: A Network Analysis Study in People With Bulimia Nervosa and With Binge Eating Disorder
}

\author{
Alessio Maria Monteleone ( $\square$ alessio.monteleone@fastwebnet.it ) \\ Universita degli Studi della Campania Luigi Vanvitelli https://orcid.org/0000-0002-6786-4458 \\ Orna Tzischinsky \\ Emek-Yezreel College \\ Giammarco Cascino \\ University of Salerno: Universita degli Studi di Salerno
}

\section{Sigal Alon}

Health care campus haifa

\section{Francesca Pellegrino}

University of Campania Luigi Vanvitelli: Universita degli Studi della Campania Luigi Vanvitelli

\section{Valeria Ruzzi}

University of Campania Luigi Vanvitelli: Universita degli Studi della Campania Luigi Vanvitelli

Yael Latzer

University of Haifa Faculty of Social Welfare and Health Sciences

\section{Research Article}

Keywords: Childhood maltreatment, emotional abuse, bulimia nervosa, binge eating disorder, network analysis, psychopathology

Posted Date: February 22nd, 2021

DOl: https://doi.org/10.21203/rs.3.rs-204849/v1

License: (c) (i) This work is licensed under a Creative Commons Attribution 4.0 International License. Read Full License

Version of Record: A version of this preprint was published at Eating and Weight Disorders - Studies on Anorexia, Bulimia and Obesity on March 28th, 2021. See the published version at https://doi.org/10.1007/s40519-021-01169-6. 


\section{Abstract}

Purpose. Childhood maltreatment (CM) experiences are associated with heightened risk of Eating Disorders (EDs). The psychopathological pathways promoting this association in people with Bulimia Nervosa (BN) and in those with Binge Eating Disorder (BED) are under-investigated.

Methods. One-hundred-eighty-one people with BN and 144 with BED filled in the Eating Disorder Inventory-2, to measure ED psychopathology, and the Childhood Trauma Questionnaire, to assess their early traumatic experiences. Network analysis was conducted in order to investigate the interplay between those variables. The shortest pathways function was employed to investigate the shortest out of all routes conveying the association between $\mathrm{CM}$ and ED core symptoms.

Results. In both people with BN and with BED, all CM types were connected to the ED psychopathology through the emotional abuse node. The association between emotional abuse and ED core symptoms (bulimia and body dissatisfaction) differed in the two groups: in people with $\mathrm{BN}$, it included ineffectiveness, while in people with BED it involved impulsivity. Interoceptive awareness, an indirect measure of emotion regulation, was included in these pathways in both groups.

Conclusion. In the light of literature showing that emotional abuse has a connecting role between $\mathrm{CM}$ and ED psychopathology also in anorexia nervosa, the present findings support the idea that emotional abuse conveys such association in all the main ED diagnoses. Ineffectiveness and impulsivity may represent the specific psychopathological dimensions connected to emotional abuse and promoting the maintenance of ED core symptoms in BN and in BED, respectively. These findings are worth of attention by clinicians.

Level of evidence: Level III: Evidence obtained from well-designed cohort or case-control analytic studies

\section{Introduction}

Eating Disorders (EDs) are complex psychiatric illnesses likely originating by the interplay of psychosocial, biological and genetic factors [1]. A recent umbrella review [2] assessing longitudinal data on pathogenetic risk factors showed that, among psychosocial factors, childhood sexual abuse and appearance-related teasing victimization are the factors more significantly associated with bulimia nervosa (BN) and any type of ED respectively. Literature cross-sectional studies have confirmed that childhood maltreatment (CM) experiences are associated with EDs. Indeed, Caslini et al. [3] suggested that childhood sexual abuse is associated with BN and Binge Eating Disorders (BED), while childhood physical abuse is associated with all types of EDs. In a more recent systematic review and meta-analysis [4], all kinds of $\mathrm{CM}$, including emotional abuse and neglect, have been found to be associated with EDs, without significant differences across the main ED diagnoses. A dose-dependent association between $\mathrm{CM}$ and symptoms severity and clinical markers of a more severe form of illness in patients with ED were also identified [4]. In this line, recent studies corroborated the association between CM and EDs from multiple levels of analyses. In particular, decreased gray matter volumes and altered connectivity between 
brain regions were detected in maltreated people with EDs when compared to both no maltreated individuals and healthy peers [5]. The functioning of the hypothalamus-pituitary-adrenal axis was impaired in terms of cortisol awakening response [6] and cortisol response to an acute social stress [7] in acute or recovered [8] maltreated ED individuals. In line with findings suggesting that CM may confer a higher vulnerability to develop ED symptoms in response to traumatic events in adulthood [9], Monteleone et al. [7] showed that ED people with CM exhibited heightened post-stress emotional arousal, body dissatisfaction and cognitive drive to restrain food intake. This evidence contributed to the possibility of identifying a maltreated ecophenotype also in people with EDs as in other psychiatric disorders [10].

Despite the importance of $\mathrm{CM}$ in EDs, the psychopathological pathways mediating the association between each CM type and ED core symptoms have been under-investigated. Emotion dysregulation [11; 12], reduced self-esteem [13] or dissociation [14] have been found as the possible mediating factors in people with anorexia nervosa (AN) or with BN. More recently, Rodgers et al. [15] indicated that depressed mood was an important driver of ED psychopathology in abused ED individuals.

Few studies have explored the association of $\mathrm{CM}$ and psychopathology in people with BED and showed either no association [16; 17] or significant association [18] with a possible mediating role of self-criticism $[19 ; 20]$. The use of different investigation methods and the assessment of different types of CM and of different outcomes (general or specific psychopathology) possibly contributed to the inconsistency of these findings. A novel method to explore the association between $\mathrm{CM}$ and ED psychopathology is the network analysis approach. This method differs from traditional statistics (i.e., multiple regression analyses) given that it identifies the connection between two variables, which points to the association between those variables taking into account the effects of all the other variables included in the network $[21 ; 22]$. Thus, network analysis simultaneously evaluates the association between each CM type and several ED psychopathology features providing a more comprehensive evaluation of this association. Furthermore, the employment of a specific network function, the shortest pathways analysis, allows to identify the shortest route between external factors, which are conditions outside the psychopathological network but not outside the person (the "external field", [21]), and the variables included in the network. We employed this method in a previous study [23] and found that all CM types were connected to ED psychopathology through emotional abuse, which in turn was connected to ED core symptoms through reduced interoceptive ability and ineffectiveness in people with restricting type AN and in patients with binge-purging symptoms, respectively. Thus, in the present study we aimed to explore the association between each CM type and ED core symptoms through the use of the shortest pathways analysis in people with BN and in those with BED. On the basis of previous studies employing this method in people with AN and with binge-purging symptoms [23] and considering that emotional abuse was the CM type more frequently associated with psychopathology in people with BED $[17 ; 18 ; 19 ; 24 ; 25]$, we hypothesized that emotional abuse should convey the association between each CM type and ED core symptoms in both study populations. In the light of literature findings [16; 19;23;25], our secondary hypothesis was that ineffectiveness mediated the association between emotional abuse and ED core symptoms. 


\section{Methods}

\section{Participants}

Consecutive patients attending the Eating Disorder Center of the Department of Psychiatry at the University of Campania "Luigi Vanvitelli" and of the Rambam Health care campus, Psychiatric division and Eating Disorder center, Haifa, Israel were recruited. Inclusion criteria for the study were: 1) current diagnosis of BN or BED, according to DSM- 5 and confirmed by the Structured Clinical Interview for DSM-5 Disorders-Research Version (SCID-RV) [26]; 2) absence of current/lifetime comorbid diagnosis of schizophrenia, bipolar disorder or substance abuse disorder; 3 ) willingness to cooperate in the experimental procedures and to sign a written informed consent.

Diagnostic assessment was made by a senior (in Israel) or trained (in Italy) psychiatrist (A.M.M.), who made the diagnosis first through a face-to-face clinical interview and then employing the SCID-RV, to confirm the ED diagnosis and psychiatric comorbidity.

\section{Procedure}

Sociodemographic and clinical data were collected as part of the routine assessment of patients with EDs.

Participants in the study were asked to complete the following questionnaires before entering specific treatment programs: 1) the Eating Disorders Inventory-2 (EDI-2) [27]; 2) the Childhood Trauma Questionnaire (CTQ) [28].

The EDI-2 [27] evaluates ED symptoms and psychopathology. The questionnaire includes 11 subscales: ineffectiveness, social insecurity, drive to thinness, interoceptive awareness, maturity fear, body dissatisfaction, perfectionism, interpersonal distrust, impulsivity, bulimia and ascetism. Cronbach's values ranged from 0.72 (maturity fear) to 0.92 (ineffectiveness).

The CTQ [28] investigates a self-report recall of childhood maltreatment experiences. It is a 28-item questionnaire which differentiates five types of $\mathrm{CM}$ : emotional neglect (EN) (cut-off score $\geq 15$; Cronbach's $a=0.89$ ), emotional abuse (EA) (cut-off score $\geq 10$; Cronbach's $a=0.84$ ), sexual abuse (SA) (cut-off score $\geq 8$; Cronbach's $a=0.86$ ), physical neglect $(P N)$ (cut-off score $\geq 8$; Cronbach's $a=0.72$ ) and physical abuse (PA) (cut-off score $\geq 8$; Cronbach's $a=0.87$ ). The cut-off scores have been defined to indicate the occurrence of each CM type [30]. To the purposes of this study, a dimensional approach was adopted by employing the sum-score of each sub-scale [29]. Indeed, the use of continuous measures to evaluate trauma is largely documented in literature studies [9] and also employed in network analysis studies [31].

The study was approved by the Institutional Board of the University of Campania L. Vanvitelli and by the Ministry of Health and the Rambam Hospital's Helsinki Ethics committee (40-09RAM). 


\section{Statistical Analysis}

Differences between people with BN and those with BED were analyzed by an independent samples t-Test and the Chi-square test, where appropriate.

Network Analysis (NA) was performed through R, version 3.4.4, using qgraph package [32]. A network is composed of nodes, which represent each variable included in the network, and edges, which are the connections among them. We have included in the network the EDI-2 sub-scores and the CTQ items. The thickness of an edge graphically represents the magnitude of the association. We have estimated partialcorrelation networks, where the association between two nodes is controlled for the influence of all other variables [32]. We have run two separate networks: one for the sample with BN and one for the BED group. In order to retain only meaningful associations, we applied a "least absolute shrinkage and selection operator" (LASSO) regularization [33], which shrinks small partial correlations and sets them to zero [34]. The Extended Bayesan Information Criterion (EBIC) [35], a parameter that sets the degree of regularization/penalty applied to sparse correlations, was set to 0.5 in these analyses. The accuracy of edge-weights was estimated by drawing bootstrapped confidence intervals calculated through "nonparametric" bootstrapping (nboots = 2500) [36]. The bootnet package [37] was used for this analysis.

Subsequently, we performed the shortest pathways analysis in each network. This kind of network detects the shortest path between two nodes, i.e., the quickest out of all the routes connecting these two nodes. The shortest path between two nodes represents the minimum number of steps needed to go from one node to the other [38], computed using Dijkstra's algorithm [39]. We employed this method in order to identify the shortest paths between each CM node, as assessed by the CTQ, and the ED core symptoms (body dissatisfaction and bulimia), evaluated by means of the EDI-2. The undirected edges assessed in this network point to conditional dependence between two variables: the edge-weight parameters reflect the strength of the associations between variables, which in turn suggests potential causal relationships $[31 ; 36]$.

\section{Results}

\section{Sample Characteristics}

Clinical characteristics of the study samples are reported in Table 1. One hundred-eighty-one people with BN and 144 people with BED were included in the study.

A current comorbid anxiety disorder was detected in 73 subjects (43 with BN and 30 with BED), while 101 individuals (51 with BN and 50 with BED) were diagnosed with a comorbid depressive disorder. The Mann-Whitney test (Table 1) showed that, compared to people with BED, people with BN reported younger age and lower BMI values and scored higher in all EDI-2 sub-scores, except for body dissatisfaction, which was higher in people with BED. CTQ emotional neglect and physical abuse scores were higher in BN people than in BED individuals. 


\section{Network Analyses}

The shortest pathways analysis identified the shortest route between the CTQ nodes and the EDI-2 core (bulimia and body dissatisfaction) symptoms. The network in Figure 1 shows the connections between each childhood trauma experience and the EDI-2 sub-scores in the group with BN. It emerged that there were no direct connections between $\mathrm{CM}$ nodes and ED symptoms, except for the connection between emotional abuse and ineffectiveness. This node was directly connected with the ED core symptom body dissatisfaction, while the shortest path to reach bulimia included the interoceptive awareness.

The network in Figure 2 illustrates the connections between childhood trauma experiences and EDI-2 in the group with BED. As in the previous network, CM nodes were not connected with ED symptoms, except for emotional abuse that was connected with impulsivity. This symptom, in turn, was positively associated with body dissatisfaction via ineffectiveness, while the shortest route to bulimia included the interoceptive awareness.

The bootstrapped confidence intervals of estimated edge-weights are reported in Supplementary Figure 1 and 2.

\section{Discussion}

The first goal of this study was to explore the connection between CM and ED psychopathology. According to our first study hypothesis, all CM types were associated to ED psychopathology through the emotional abuse experience in both people with BN and those with BED. These populations, instead, differed in relation to the second study hypothesis. Indeed, in the BN group ineffectiveness and interoceptive awareness were included in shortest pathway between emotional abuse and core symptoms; in the BED group, also impulsivity was included in that pathway and was directly connected to emotional abuse.

The employment of the shortest pathways analysis allowed us to highlight the central role of emotional abuse in the association between CM and ED psychopathology. Indeed, we found that all CM types convey their effects on ED psychopathology through emotional abuse. This kind of early abuse refers to experiences of being humiliated or receiving self-demeaning behaviors [28]. Bullying or appearancerelated teasing experiences as well as physical or sexual abuse events may promote emotional abuse. These findings confirm the importance to evaluate childhood emotional abuse when assessing CM in people with $\mathrm{BN}$ or with $\mathrm{BED}$, despite sexual abuse has been longitudinally recognized as the unique risk factor for EDs [2] and despite literature highlighting the association of physical and sexual abuse with EDs [3]. In this line, emotional abuse was found to be the only CM type directly associated to ED psychopathology, without any effect of psychiatry comorbidity [40] and previous studies suggested that different types of CM do not have the same impact on ED symptoms [41; 42]. Furthermore, in people with $\mathrm{AN}$ and in those with $\mathrm{BN}$, the emotional trauma was the specific CM type contributing to the heightened emotional stress reactivity and to the increased food related and body concerns in response to a social stress test [7]. In people with BED, several studies have found that emotional abuse was higher than in 
obese people without BED [18] and was the unique CM type associated to psychopathology $[17 ; 18 ; 19 ;$ 25; 43]. However, none of these studies assessed the association between emotional abuse and psychopathology taking into account the effects of the other CM types and the covariation among symptoms, as done in the present network analysis. Only one previous study [23] has employed the same method in patients with restricting type AN and in patients with binge-purging symptoms and has revealed that emotional abuse was the node conveying the effects of each CM experience on ED psychopathology. Taken together, these results point to emotional abuse as the $\mathrm{CM}$ type promoting the association of $\mathrm{CM}$ and psychopathology across all the principal ED diagnoses.

The second main finding of this study is that ineffectiveness and interoceptive awareness are included in the shortest pathways between emotional abuse and ED core symptoms in people with BN, while also impulsivity was involved in these pathways in individuals with BED. In people with BN, ineffectiveness was directly associated with body dissatisfaction and was associated to bulimic symptoms through the interoceptive awareness node. According to the EDI-2 questionnaire [27], we found that inadequacy, loneliness and empty feelings may be associated with lower accuracy to detect and regulate inner and emotional states, which in turn are associated with bulimic symptoms. Although causality cannot be inferred, the observed pathway is consistent with models describing ED behaviors as maladaptive strategies to cope with emotions [44; 45]. Furthermore, these findings are in line with those detected in AN or BN patients with binge-purging symptoms [23] and with literature data highlighting the role of ineffectiveness as mediating the association between CM and ED psychopathology in BN [13].

Similarly to people with $\mathrm{BN}$, in those with BED ineffectiveness was connected to body dissatisfaction. However, in the BED group, impulsivity instead of ineffectiveness was connected to emotional abuse. Thus, the connection between emotional abuse and impulsivity may represent a specific pathway promoting psychopathology in people with BED, while the same role was played by ineffectiveness in BN and by interoceptive awareness in people with AN restricting type [23]. Impulsivity is a multidimensional personality trait spanning a cognitive component named reward sensitivity, a behavioral dimension related to inhibition and an affective component [46; 47]. The BED has been conceptualized as a phenotype within the obese spectrum characterized by heightened impulsivity $[48 ; 49 ; 50]$ with increased rash-spontaneous behavior in general and, specifically, towards food [51]. Furthermore, impulsivity is associated with negative urgency [52], that is the need to regulate negative emotions engaging in rash actions and risky behaviors (i.e., impulsivity) [53], especially in ED patients with food addiction [54]. This suggests that bulimic ingestion of food can be conceived as an attempt to escape unbearable emotions $[54 ; 55]$. This theory may be supported by our network pathway showing a connection between impulsivity, lower interoceptive awareness and bulimic symptoms. Furthermore, the association of this pattern with emotional abuse is in line with studies showing that impulsivity mediates the association between childhood abuse and disordered eating in a non-clinical population [56].

Limitations of this study need to be acknowledged. First, the cross-sectional nature of these data does not make possible to infer causality regarding the connections identified in the networks. Second, the CTQ provides a self-report assessment of childhood adverse experiences, that is possibly affected by 
recalling bias, without characterization of the specific nature of the trauma or the period when it occurred. Third, the lack of inclusion in the network of general psychiatric symptoms, which may be advisable in the light of high rate of psychiatry comorbidity in people with BN and with BED [1;48], precludes a complete assessment of the connections between $\mathrm{CM}$ and the whole psychopathology of BN and BED.

The main strength of this study is that it allows to explore, for the first time in people with BN and with $B E D$, the associations between different CM types and ED symptoms taking into account for the effects of each CM type and for covariation between symptoms and showing the shortest out of all routes between $\mathrm{CM}$ and $\mathrm{ED}$ symptoms.

\section{Conclusion}

Emotional abuse represents the $\mathrm{CM}$ experience that promotes the association of early adverse events with ED psychopathology in people with AN [23], with BN or with BED. Thus, emotional abuse may be conceptualized as a possible common mediator through which all CM types converge on ED psychopathology in all principal ED groups. This should prompt clinicians as well as researchers to investigate the childhood emotional abuse experiences in people with EDs. However, specific ED psychopathological variables seem to connect emotional abuse and ED symptoms in each ED group: interoceptive awareness in people with AN [23]; ineffectiveness in those with BN and impulsivity in those with BED. The network theory [17] suggests that the interplay between nodes may maintain a state of prolonged activations of further symptoms in the network. Therefore, these specific pathways may be taken into account in treatment and prevention programs. Reduced self-esteem and high impulsivity are central factors in the psychopathology of BN and of BED [48; 57]: these findings suggest clinicians to evaluate their connections with early traumatic experiences and with trauma related maladaptive schemas and to address these connections in psychotherapy interventions.

\section{What is already known on this subject?}

All kinds of childhood maltreatment are associated with an increased risk to develop an Eating Disorder. People with Eating Disorders and history of childhood maltreatment show a more severe form of the illness. The pathways promoting the association between childhood maltreatment and eating disorder psychopathology have been under-investigated.

\section{What does this study add?}

In people with bulimia nervosa and with binge eating disorder all types of childhood maltreatment are associated to psychopathology through the emotional abuse experience. The shortest pathways conveying the association between emotional abuse and eating disorder core symptoms include ineffectiveness in bulimia nervosa and impulsivity in binge eating disorder.

\section{Declarations}




\section{Funding}

This research did not receive any specific grant from funding agencies in the public, commercial, or notfor-profit sectors.

\section{Conflicts of interest}

None.

\section{Availability of data and material}

The data that support the findings of this study are available from the corresponding author upon reasonable request.

\section{Ethics Approval}

The study was approved by the Institutional Board of the University of Campania L. Vanvitelli and by the Ministry of Health and the Rambam Hospital's Helsinki Ethics committee.

\section{Authors' Contributions}

Conceptualization: A.M. Monteleone, O. Tzischinsky, Y. Latzer; Methodology: G. Cascino, A.M. Monteleone, Y. Latzer; Formal analysis and investigation: G. Cascino, V. Ruzzi, F. Pellegrino, S. Alon; Writing - original draft preparation: A.M. Monteleone; Writing - review and editing: A.M. Monteleone, 0. Tzischinsky, Y. Latzer.

\section{Acknowledgements}

None.

\section{References}

1. Treasure J, Duarte TA, Schmidt U (2020) Eating disorders. Lancet 395:899-911

2. Solmi M, Radua J, Stubbs B et al (2020) Risk factors for eating disorders: an umbrella review of published meta-analyses. Brazilian J Psychiatry. doi:10.1590/1516-4446-2020-1099

3. Caslini M, Bartoli F, Crocamo C et al (2016) Disentangling the Association Between Child Abuse and Eating Disorders. Psychosom Med 78:79-90. doi:10.1097/PSY.0000000000000233

4. Molendijk ML, Hoek HW, Brewerton TD, Elzinga BM (2017) Childhood maltreatment and eating disorder pathology: a systematic review and dose-response meta-analysis. Psychol Med 47:14021416. doi:10.1017/S0033291716003561

5. Monteleone AM, Monteleone P, Esposito F et al (2017) The effects of childhood maltreatment on brain structure in adults with eating disorders. World J Biol Psychiatry 1-10. doi:10.1080/15622975.2017.1395071 
6. Monteleone AM, Monteleone P, Volpe U et al (2018) Impaired cortisol awakening response in eating disorder women with childhood trauma exposure: Evidence for a dose-dependent effect of the traumatic load. Psychol Med 48:. doi:10.1017/S0033291717002409

7. Monteleone AM, Cascino G, Ruzzi V et al (2020) Emotional traumatic experiences significantly contribute to identify a maltreated ecophenotype sub-group in eating disorders: Experimental evidence. Eur Eat Disord Rev erv.2818. doi:10.1002/erv.2818

8. Lelli L, Castellini G, Cassioli E et al (2019) Cortisol levels before and after cognitive behavioural therapy in patients with eating disorders reporting childhood abuse: A follow-up study. Psychiatry Res 275:269-275. doi:10.1016/j.psychres.2019.03.046

9. Trottier K, MacDonald DE (2017) Update on Psychological Trauma, Other Severe Adverse Experiences and Eating Disorders: State of the Research and Future Research Directions. Curr Psychiatry Rep 19:45. doi:10.1007/s11920-017-0806-6

10. Teicher MH, Samson JA (2013) Childhood Maltreatment and Psychopathology: A Case for Ecophenotypic Variants as Clinically and Neurobiologically Distinct Subtypes. Am J Psychiatry 170:1114-1133. doi:10.1176/appi.ajp.2013.12070957

11. Racine SE, Wildes JE (2015) Emotion dysregulation and anorexia nervosa: an exploration of the role of childhood abuse. Int J Eat Disord 48:55-58. doi:10.1002/eat.22364

12. Mills P, Newman EF, Cossar J, Murray G (2015) Emotional maltreatment and disordered eating in adolescents: Testing the mediating role of emotion regulation. Child Abus Negl 39:156-166. doi:10.1016/j.chiabu.2014.05.011

13. Groleau P, Steiger H, Bruce K et al (2012) Childhood emotional abuse and eating symptoms in bulimic disorders: An examination of possible mediating variables. Int J Eat Disord 45:326-332. doi:10.1002/eat.20939

14. Moulton SJ, Newman E, Power K et al (2015) Childhood trauma and eating psychopathology: A mediating role for dissociation and emotion dysregulation? Child Abus Negl 39:167-174. doi:10.1016/j.chiabu.2014.07.003

15. Rodgers RF, DuBois R, Thiebaut S et al (2019) Structural Differences in Eating Disorder Psychopathology After History of Childhood Abuse: Insights From a Bayesian Network Analysis. J Abnorm Psychol. doi:10.1037/abn0000470

16. Becker DF, Grilo CM (2011) Childhood maltreatment in women with binge-eating disorder: Associations with psychiatric comorbidity, psychological functioning, and eating pathology. In: Eating and Weight Disorders. Springer International Publishing, p e113

17. Grilo CM, Masheb RM (2001) Childhood psychological, physical, and sexual maltreatment in outpatients with binge eating disorder: Frequency and associations with gender, obesity, and eatingrelated psychopathology. Obes Res 9:320-325. doi:10.1038/oby.2001.40

18. Amianto F, Spalatro AV, Rainis M et al (2018) Childhood emotional abuse and neglect in obese patients with and without binge eating disorder: Personality and psychopathology correlates in adulthood. Psychiatry Res 269:692-699. doi:10.1016/j.psychres.2018.08.089 
19. Dunkley DM, Masheb RM, Grilo CM (2010) Childhood maltreatment, depressive symptoms, and body dissatisfaction in patients with binge eating disorder: The mediating role of self-criticism. Int $\mathrm{J}$ Eat Disord 43:274-281. doi:10.1002/eat.20796

20. Feinson MC, Hornik-Lurie T (2016) "Not good enough:" Exploring self-criticism's role as a mediator between childhood emotional abuse \& adult binge eating. Eat Behav 23:1-6. doi:10.1016/j.eatbeh.2016.06.005

21. Borsboom D (2017) A network theory of mental disorders. World Psychiatry 16:5-13. doi:10.1002/wps.20375

22. Fried El, van Borkulo CD, Cramer AOJ et al (2017) Mental disorders as networks of problems: a review of recent insights. Soc Psychiatry Psychiatr Epidemiol 52:1-10. doi:10.1007/s00127-016-1319-z

23. Monteleone AM, Cascino G, Pellegrino F et al (2019) The association between childhood maltreatment and eating disorder psychopathology: A mixed-model investigation. Eur Psychiatry 61:111-118. doi:10.1016/j.eurpsy.2019.08.002

24. Allison KC, Grilo CM, Masheb RM, Stunkard AJ (2007) High self-reported rates of neglect and emotional abuse, by persons with binge eating disorder and night eating syndrome. Behav Res Ther 45:2874-2883. doi:10.1016/j.brat.2007.05.007

25. Latzer Y, Rozenstain-Hason M, Kabakov $O$ et al (2020) Childhood maltreatment in patients with binge eating disorder with and without night eating syndrome vs. control. Psychiatry Res 293:. doi:10.1016/j.psychres.2020.113451

26. First MB, Williams JBW, Karg RS, Spitzer RL User's Guide to Structured Clinical Interview for Dsm-5 Disorders (Scid-5-cv): Clinician Version Title: User's Guide to Structured Clinical

27. Garner D (1991) Eating disorder inventory-2. Odessa, FL: Psychological Assessment Resources. https//:doi.org/10.1177/ 1073191111428761

28. Bernstein DP, Stein JA, Newcomb MD et al (2003) Development and validation of a brief screening version of the Childhood Trauma Questionnaire. Child Abuse Negl 27:169-190. doi:10.1016/S01452134(02)00541-0

29. Bernstein DP, Fink L (1998) Childhood trauma questionnaire: a retrospective self-report : manual. Harcourt Brace \& Co, Orlando

30. Walker EA, Gelfand A, Katon WJ et al (1999) Adult health status of women with histories of childhood abuse and neglect. Am J Med 107:332-339. doi:10.1016/S0002-9343(99)00235-1

31. Epskamp S, Cramer AOJ, Waldorp LJ et al (2012) qgraph: Network Visualizations of Relationships in Psychometric Data. J Stat Softw 48:1-18. doi:10.18637/jss.v048.i04

32. Epskamp S, Fried El (2018) A tutorial on regularized partial correlation networks. Psychol Methods 23:617-634. doi:10.1037/met0000167

33. Chen J, Chen Z (2008) Extended Bayesian information criteria for model selection with large model spaces. Biometrika 95:759-771. doi:10.1093/biomet/asn034 
34. McNally RJ (2016) Can network analysis transform psychopathology? Behav Res Ther 86:95-104. doi:10.1016/j.brat.2016.06.006

35. Epskamp S, Borsboom D, Fried El (2018) Estimating psychological networks and their accuracy: A tutorial paper. Behav Res Methods 50:195-212. doi:10.3758/s13428-017-0862-1

36. Epskamp S, Maris GKJ, Waldorp LJ, Borsboom D (2016) Network Psychometrics. Wiley Handb Psychom Test A Multidiscip Ref. Surv Scale Test Dev 2-2:953-986

37. Brandes U (2008) On variants of shortest-path betweenness centrality and their generic computation. Soc Networks 30:136-145. doi:10.1016/J.SOCNET.2007.11.001

38. Dijkstra EW (1959) A note on two problems in connexion with graphs. Numerische mathematik 1(1):269-271

39. Isvoranu A-M, van Borkulo CD, Boyette L-L et al (2017) A Network Approach to Psychosis: Pathways Between Childhood Trauma and Psychotic Symptoms. Schizophr Bull 43:187-196. doi:10.1093/schbul/sbw055

40. Guillaume S, Jaussent I, Maimoun L et al (2016) Associations between adverse childhood experiences and clinical characteristics of eating disorders. Sci Rep 6:35761. doi:10.1038/srep35761

41. Kent A, Waller G, Dagnan D (1999) A Greater role of emotional than physical or sexual abuse in predicting disordered eating attitudes: The role of mediating variables. Int J Eat Disord 25:159-167. doi: 10.1002/(SICI)1098-108X(199903)25:2<159::AID-EAT5>3.0.CO;2-F

42. Monteleone AM, Ruzzi V, Patriciello G et al (2019) Parental bonding, childhood maltreatment and eating disorder psychopathology: an investigation of their interactions. Eat Weight Disord - Stud Anorexia, Bulim Obes. doi: 10.1007/s40519-019-00649-0

43. Grilo CM, Masheb RM (2002) Childhood maltreatment and personality disorders in adult patients with binge eating disorder. Acta Psychiatr Scand 106:183-188. doi:10.1034/j.16000447.2002.02303.x

44. Rieger E, Van Buren DJ, Bishop M et al (2010) An eating disorder-specific model of interpersonal psychotherapy (IPT-ED): Causal pathways and treatment implications. Clin Psychol Rev 30:400-410. doi:10.1016/j.cpr.2010.02.001

45. Lavender JM, Wonderlich SA, Engel SG et al (2015) Dimensions of emotion dysregulation in anorexia nervosa and bulimia nervosa: A conceptual review of the empirical literature. Clin Psychol Rev 40:111-122. doi:10.1016/J.CPR.2015.05.010

46. Dawe S, Loxton NJ (2004) The role of impulsivity in the development of substance use and eating disorders. Neurosci Biobehav Rev 28:343-351

47. Sharma L, Markon KE, Clark LA (2014) Toward a theory of distinct types of "impulsive" behaviors: A meta-analysis of self-report and behavioral measures. Psychol Bull 140:374-408. doi:10.1037/a0034418

48. Agüera Z, Lozano-Madrid M, Mallorquí-Bagué $\mathrm{N}$ et al (2020) A review of binge eating disorder and obesity. Neuropsychiatrie 1-11 
49. Gerlach G, Herpertz S, Loeber S (2015) Personality traits and obesity: A systematic review. Obes Rev 16:32-63. doi:10.1111/obr.12235

50. Kessler RM, Hutson PH, Herman BK, Potenza MN (2016) The neurobiological basis of binge-eating disorder. Neurosci Biobehav Rev 63:223-238

51. Giel KE, Teufel M, Junne F et al (2017) Food-related impulsivity in obesity and binge eating disordera systematic update of the evidence. Nutrients 9

52. Lee-Winn AE, Townsend L, Reinblatt SP, Mendelson T (2016) Associations of neuroticism-impulsivity and coping with binge eating in a nationally representative sample of adolescents in the United States. Eat Behav 22:133-140. doi:10.1016/j.eatbeh.2016.06.009

53. Racine SE, VanHuysse JL, Keel PK et al (2017) Eating disorder-specific risk factors moderate the relationship between negative urgency and binge eating: A behavioral genetic investigation. $J$ Abnorm Psychol 126:481-494. doi:10.1037/abn0000204

54. Wolz I, Hilker I, Granero R et al (2016) "Food Addiction" in Patients with Eating Disorders is Associated with Negative Urgency and Difficulties to Focus on Long-Term Goals. Front Psychol 7:61. doi:10.3389/fpsyg.2016.00061

55. Troisi A (2020) Bariatric Surgery and Mental Health. In: Bariatric Psychology and Psychiatry. Springer International Publishing, pp 1-12

56. Dworkin E, Javdani S, Verona E, Campbell R (2014) Child sexual abuse and disordered eating: The mediating role of impulsive and compulsive tendencies. Psychol Violence 4:21-36. doi:10.1037/a0031779

57. Solmi M, Collantoni E, Meneguzzo P et al (2018) Network analysis of specific psychopathology and psychiatric symptoms in patients with eating disorders. Int J Eat Disord 51:680-692. doi:10.1002/eat.22884

\section{Tables}


Table 1

Clinical characteristics of the diagnostic groups.

\begin{tabular}{|c|c|c|c|c|}
\hline & People with BN $(n=181)$ & $\begin{array}{l}\text { People with BED } \\
(n=144)\end{array}$ & Mann-Whitney & $\mathbf{p}$ \\
\hline Age & $28.41 \pm 9.31$ & $37.33 \pm 13.59$ & 14128.5 & $<0.001$ \\
\hline Age at Onset & $18.81 \pm 5.78$ & $23.23 \pm 12.07$ & 10128.0 & 0.05 \\
\hline Illness Duration, yrs & $9.54 \pm 8.31$ & $13.15 \pm 10.74$ & 10341.0 & 0.01 \\
\hline Body Mass Index & $23.66 \pm 6.18$ & $35.86 \pm 8.42$ & 19786.0 & $<0.001$ \\
\hline $\begin{array}{l}\text { EDI-2 } \\
\text { Ineffectiveness } \\
\text { Maturity Fear } \\
\text { Social Insecurity } \\
\text { Body Dissatisfaction } \\
\text { Perfectionism } \\
\text { Interpersonal Distrust } \\
\text { Impulsivity } \\
\text { Drive for Thinness } \\
\text { Bulimia } \\
\text { Interoceptive Awareness } \\
\text { Asceticism }\end{array}$ & $\begin{array}{l}12.85 \pm 7.89 \\
8.75 \pm 5.72 \\
7.71 \pm 4.30 \\
16.02 \pm 7.25 \\
7.23 \pm 4.60 \\
6.77 \pm 4.81 \\
9.86 \pm 7.22 \\
15.55 \pm 5.27 \\
10.58 \pm 5.64 \\
14.02 \pm 6.89 \\
9.23 \pm 4.46\end{array}$ & $\begin{array}{l}9.58 \pm 7.32 \\
6.68 \pm 5.02 \\
5.24 \pm 3.31 \\
18.88 \pm 6.13 \\
6.03 \pm 4.43 \\
4.97 \pm 4.33 \\
5.43 \pm 5.17 \\
12.81 \pm 5.63 \\
8.13 \pm 5.18 \\
8.54 \pm 6.12 \\
6.61 \pm 3.68\end{array}$ & $\begin{array}{l}10040.5 \\
10458.5 \\
8697.5 \\
16325.0 \\
11247.0 \\
10318.5 \\
8350.0 \\
9225.5 \\
9.971 .0 \\
7398.0 \\
8777.0\end{array}$ & $\begin{array}{l}<0.001 \\
<0.001 \\
<0.001 \\
<0.001 \\
0.01 \\
<0.001 \\
<0.001 \\
<0.001 \\
<0.001 \\
<0.001 \\
<0.001\end{array}$ \\
\hline $\begin{array}{l}\text { CTQ } \\
\text { Emotional Neglect } \\
\text { Emotional Abuse } \\
\text { Sexual Abuse } \\
\text { Physical Neglect } \\
\text { Physical Abuse }\end{array}$ & $\begin{array}{l}13.30 \pm 4.83 \\
10.21 \pm 4.71 \\
6.69 \pm 3.61 \\
7.28 \pm 2.51 \\
6.89 \pm 3.08\end{array}$ & $\begin{array}{l}11.76 \pm 4.90 \\
9.96 \pm 4.47 \\
6.55 \pm 3.34 \\
6.97 \pm 2.21 \\
6.25 \pm 2.51\end{array}$ & $\begin{array}{l}10838.0 \\
12970.0 \\
12973.5 \\
12632.0 \\
11718.0\end{array}$ & $\begin{array}{l}<0.01 \\
0.71 \\
0.85 \\
0.43 \\
<0.05\end{array}$ \\
\hline
\end{tabular}

\section{Figures}




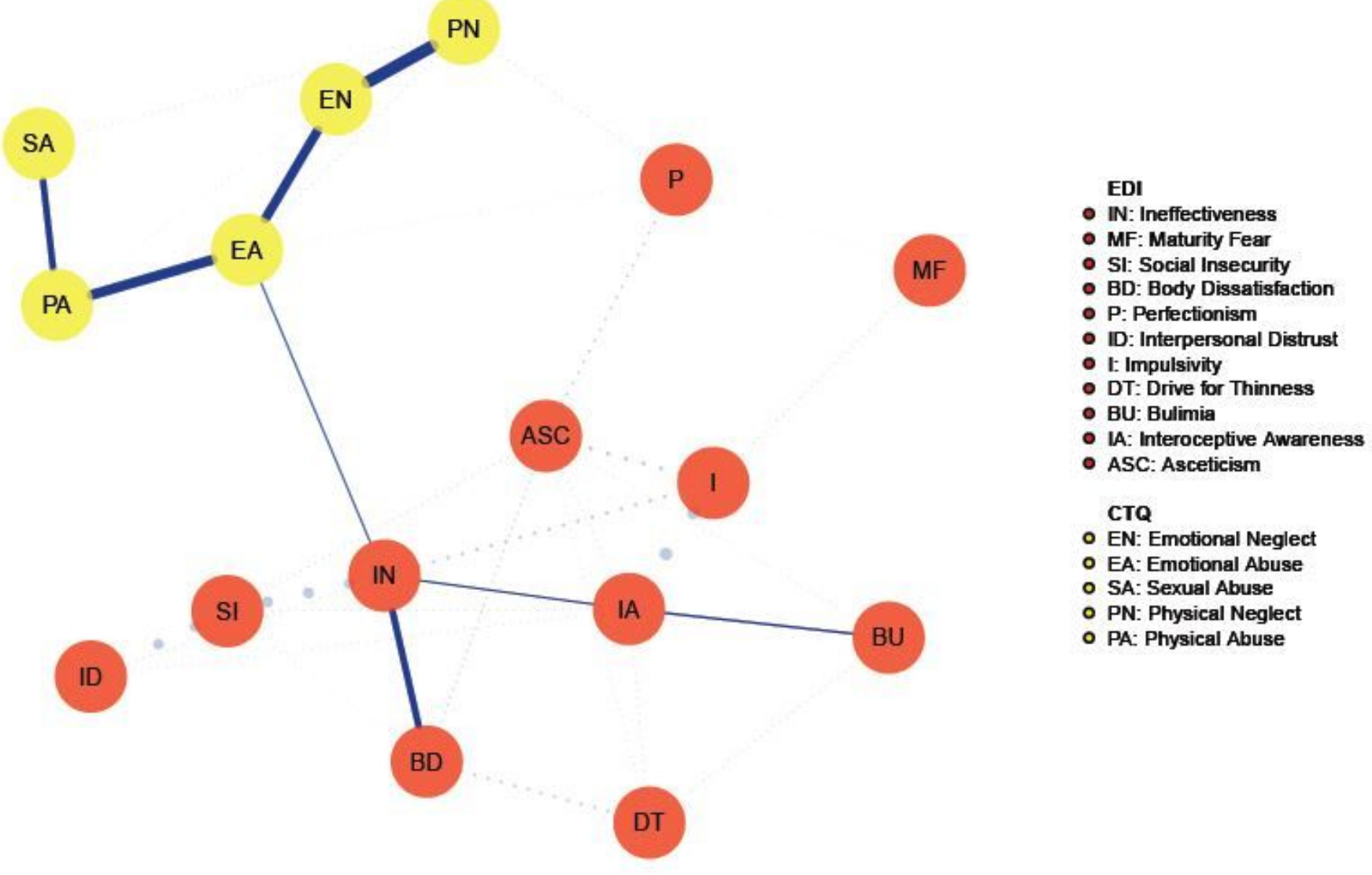

Figure 1

Shortest Pathways Network in the bulimia nervosa group Network illustrating the shortest path between childhood maltreatment dimensions and eating disorders symptoms in the bulimia nervosa group. For color version, see this figure online. 


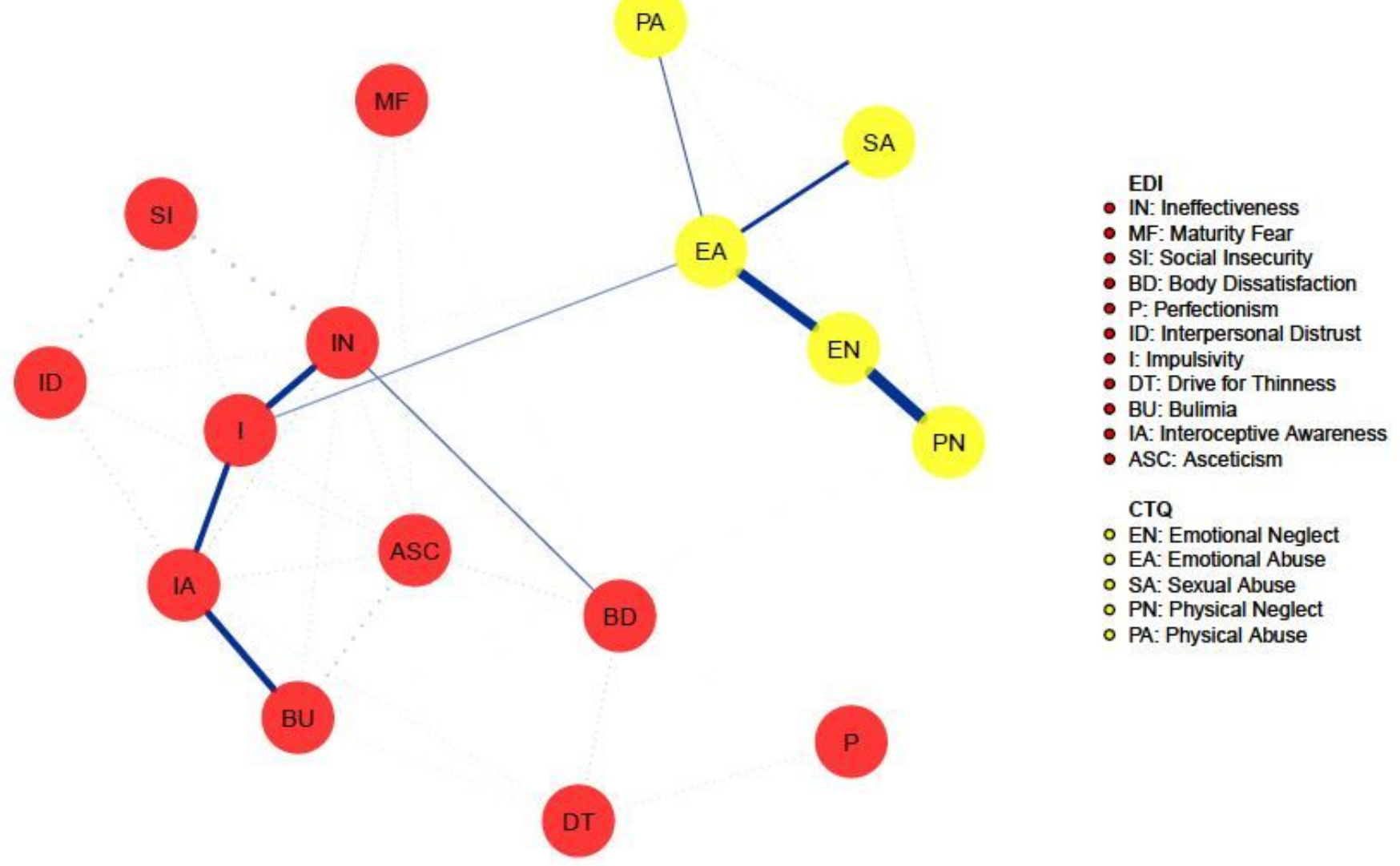

Figure 2

Shortest Pathways Network in the binge eating disorder group Network illustrating the shortest path between childhood maltreatment dimensions and eating disorders symptoms in the binge eating disorder group. For color version, see this figure online.

\section{Supplementary Files}

This is a list of supplementary files associated with this preprint. Click to download.

- $S$

- $S$ 